\title{
ON RAMSEY-TURÁN TYPE THEOREMS FOR HYPERGRAPHS
}

\author{
P. ERDŐS and Vera T. SÓS \\ Dedicated to Tibor Gallai on his seventieth birthday
}

Received 3 June 1982

Let $H^{r}$ be an $r$-uniform hypergraph. Let $g=g\left(n ; H^{r}\right)$ be the minimal integer so that any $r$-uniform hypergraph on $n$ vertices and more than $g$ edges contains a subgraph isomorphic to $H^{r}$. Let $e=f\left(n ; H^{r}, \varepsilon n\right)$ denote the minimal integer such that every $r$-uniform hypergraph on $n$ vertices with more than $e$ edges and with no independent set of $\varepsilon n$ vertices contains a subgraph isomorphic to $\mathrm{H}^{r}$.

We show that if $r>2$ and $H^{r}$ is e.g. a complete graph then

$$
\lim _{\varepsilon \rightarrow 0} \lim _{n \rightarrow \infty}\left(\begin{array}{l}
n \\
r
\end{array}\right)^{-1} f\left(n ; H^{r}, \varepsilon n\right)=\lim _{n \rightarrow \infty}\left(\begin{array}{l}
n \\
r
\end{array}\right)^{-1} g\left(n ; H^{r}\right)
$$

while for some $H^{r}$ with $\lim _{n \rightarrow \infty}\left(\begin{array}{l}n \\ r\end{array}\right)^{-1} g\left(n ; H^{r}\right) \neq 0$

$$
\lim _{\varepsilon \rightarrow 0} \lim _{n \rightarrow \infty}\left(\begin{array}{l}
n \\
r
\end{array}\right)^{-1} f\left(n ; H^{r}, \varepsilon n\right)=0 .
$$

This is in strong contrast with the situation in case $r=2$. Some other theorems and many unsolved problems are stated.

Let $H^{r}(V ; E)$ be an $r$-uniform hypergraph and $f\left(n ; H^{r}\right)$ be the smallest integer for which every $r$-uniform hypergraph of $n$ vertices and more than $f\left(n ; H^{r}\right)$ edges contains a subgraph isomorphic to $H^{r}$. A $G_{n}^{r}(V ; E)$ is called an extremal graph belonging to $H^{r}$, if $|V|=n, e\left(G_{n}^{r}\right)=f\left(n ; H^{r}\right)$ and $G_{n}^{r}$ does not contain a subgraph isomorphic to $H^{r}$ (e(..) denotes the number of hyperedges).

The determination (or estimation) of $f\left(n ; H^{r}\right)$ is the fundamental problem of extremal graph theory which was started by Turán [9]. As a generalization of Turán's theorem, the well-known Erdös-Stone theorem [6] states the following.

For an arbitrary $\mathrm{H}^{2}$ :

$$
f\left(n ; H^{2}\right)=\left(\frac{1}{2} \frac{\chi\left(H^{2}\right)-2}{\chi\left(H^{2}\right)-1}+o(1)\right) n^{2} \quad \text { if } \quad n \rightarrow \infty
$$

where $\chi(H)$ is the chromatic number of $H$.

AMS subject classification (1980): 05 C 65;05 C 35, 05 C 55. 
First of all, we remark that for $r>2$ almost nothing is known about $f\left(n ; H^{r}\right)$. E.g. for the simplest graphs $K_{4}^{3}$ (the complete 3-uniform hypergraph on 4 vertices) or $H^{3}(4 ; 3)$ (three triples on four vertices) not even the asymptotic value of $f\left(n ; H^{3}\right)$ is known. Turán's classical conjecture is, that

$$
f\left(n ; K_{4}^{3}\right) \sim \frac{5}{9}\left(\begin{array}{l}
n \\
3
\end{array}\right) \quad \text { if } n \rightarrow \infty
$$

and it is very probably that

$$
f\left(n ; H^{3}(4 ; 3)\right) \sim \frac{1}{4}\left(\begin{array}{l}
n \\
3
\end{array}\right) \text { if } n \rightarrow \infty
$$

As to the general case, it is easy to see that for an arbitrary $H^{r}$

$$
\lim _{n \rightarrow \infty}\left(\begin{array}{l}
n \\
r
\end{array}\right)^{-1} f\left(n ; H^{r}\right)=c\left(H^{r}\right)
$$

exists. It is well-known [4] that $c\left(H^{r}\right)=0$ if and only if the vertices of $H^{r}$ can be split into $r$ classes so that every edge of $H^{r}$ meets all $r$ classes.

We observed [5], [7] that for $r=2, H=K_{k}$ (where $K_{k}$ is the complete graph on $k$ vertices), the extremal graph is stable in the following sense: it contains "very large" independent sets and if we put on a condition which decreases the size of the maximal independent set in $G_{n}$ then the number of edges of the corresponding extremal graphs gets drastically reduced. More precisely, let $f\left(n ; H^{r}, l\right)$ be the smallest integer for which every graph of $n$ vertices and more than $f\left(n ; H^{r}, l\right)$ edges either contains a subgraph isomorphic to $H^{r}$ or it contains an independent set of size $l$.

Due to Ramsey's theorem for fixed $H^{r}$ and $l, f\left(n ; H^{r}, l\right)=0$ if $n>R\left(H^{r}, l\right)$. Therefore, the problem makes sense only in the case when either $\left|V\left(H^{r}\right)\right| \rightarrow \infty$ or $l \rightarrow \infty$. Referring to the case $r=2$ and $H=K_{k}$, we proved

$$
\lim _{\varepsilon \rightarrow 0} \lim _{n \rightarrow \infty}\left(\begin{array}{l}
n \\
2
\end{array}\right)^{-1} f\left(n ; K_{k}, \varepsilon n\right)=\left\{\begin{array}{llll}
\frac{k-3}{(k-1)} & \text { if } \quad k & \text { odd } \\
\frac{3 k-10}{(3 k-4)} & \text { if } \quad k & \text { even }
\end{array}\right.
$$

while by Turán's theorem

$$
\lim _{n \rightarrow \infty}\left(\begin{array}{l}
n \\
2
\end{array}\right)^{-1} f\left(n ; K_{k}\right)=\frac{k-2}{(k-1)}
$$

(For $k$ odd see [5], for $k=2$ see [1], [8] and for $k>2$, even see [2].)

In this paper, we investigate analogous problems for hypergraphs. The main result of this paper is that surprisingly the situation is quite different for hypergraphs. E.g. for $K_{k}^{r}$ (and for a more general class of graphs) the condition that the largest independent set has size $o(n)$ does not change the situation. We prove

Theorem 1. Let $r \geqq 3, H^{r}$ be an $r$-uniform hypergraph, $E=\left\{h_{1}, \ldots, h_{m}\right\}$ be the edge-set of $H^{r}$. Suppose $H^{r}$ satisfies the condition

$$
\text { for every } i, 1 \leqq i \leqq m \text { there exist a } j \neq i \text { such that }\left|h_{i} \cap h_{j}\right| \geqq 2 \text {. }
$$


Let $\lim _{n \rightarrow \infty}\left(\begin{array}{l}n \\ r\end{array}\right)^{-1} f\left(n ; H^{r}\right)=c\left(H^{r}\right)$ and $\lim _{\varepsilon \rightarrow 0} \lim _{n \rightarrow \infty}\left(\begin{array}{l}n \\ r\end{array}\right)^{-1} f\left(n ; H^{r}, \varepsilon n\right)=c^{*}\left(H^{r}\right)$. Then

$$
c^{*}\left(H^{r}\right)=c\left(H^{r}\right) \text {. }
$$

Remark. Condition (7) holds e.g. for $K_{k}^{r}$ and also for $H^{3}$ (4.3).

Proof. Our idea in the proof is that if there are large independent sets in the extremal graph, we spoil them by adding not too many new edges and then we have to omit some, but not too many, to destroy the possible $H$ 's and not to create large independent sets.

Let $\alpha\left(G^{r}\right)$ denote the size of the largest independent set of $G^{r}$. We use the following theorem of Erdôs-Hajnal [3]: properties:

For arbitrary $\eta>0$ and $m>N(\eta)$ there exists a graph $L_{m}^{r}$ with the following

$$
\left\{\begin{array}{l}
e\left(L_{m}^{r}\right)<m^{3 / 2}, \\
\alpha\left(L_{m}^{r}\right)<\eta m, \\
\text { if } e_{i}, e_{j} \in E\left(L_{m}^{r}\right), \quad i \neq j, \text { then }\left|e_{i} \cap e_{j}\right| \leqq 1 .
\end{array}\right.
$$

Let $\delta>0$ and $\varepsilon>0$ be arbitrary and $\left(G_{n}^{r}\right)$ be a graph satisfying

$$
\begin{gathered}
H^{r} \nsubseteq G_{n}^{r} \\
\varepsilon n>N\left(\varepsilon^{2}\right) \\
e\left(G_{n}^{r}\right)>(c-\delta)\left(\begin{array}{l}
n \\
3
\end{array}\right)
\end{gathered}
$$

where $c=c\left(H^{r}\right)$. Decompose the vertex set $V=V\left(G_{n}^{r}\right)$ in the form

$$
V=B \cup \bigcup_{i=1}^{k} A_{i}
$$

where $\alpha\left(G^{r}(B ; E(B))<\frac{\varepsilon n}{2}\right.$ and

$$
\alpha\left(G^{r}\left(A_{i} ; E\left(A_{i}\right)\right)>\frac{\varepsilon n}{2} .\right.
$$

Obviously, $k \leqq 2 / \varepsilon$. We place into the set $A_{i}$ a hypergraph $L^{r}(i)$ with $V\left(L^{r}(i)\right)=A_{i}$ and which satisfies (*) with $\eta=\varepsilon^{2}$. So we added to our $G_{n}^{r}$ new edges and the new enlarged hypergraph has clearly no independent set of size $>\varepsilon n$. But this new graph may contain a graph isomorphic to $H^{r}$. To avoid this, omit all edges $e \in E\left(G_{n}^{r}\right)$ which intersect any of our new edges in at least two vertices. So we omitted at most $O\left(n^{r-\frac{1}{2}}\right)$ edges. Observe that this final graph $G_{n}^{* r}$

(A) contains no isomorphic copy of $H^{r}$ due to the condition on $H^{r}$.

(B) $\alpha\left(G_{n}^{* r}\right)<\varepsilon n$ since we did not omit any edge contained in any of the $A_{i}$, $1 \leqq i \leqq k$.

$$
\left|E\left(G_{n}^{* r}\right)\right|>(c-\delta)\left(\begin{array}{l}
n \\
r
\end{array}\right)+O\left(n^{r-\frac{1}{2}}\right)
$$

Since $\delta>0$ was arbitrary, the proof of Theorem 1 is complete. 
On the other hand we state

Theorem 2. Let $H^{r}$ be a graph for which there is a partition of the vertex set

so that

$$
V\left(H^{r}\right)=\bigcup_{i=1}^{r} A_{i}
$$

and

$$
E\left(H^{r}\right)=E_{1} \cup E_{2}
$$

$$
\left|h \cap A_{i}\right|=1 \quad \text { for } \quad i=1, \ldots, r \quad \text { if } \quad h \in E_{1},
$$

furthermore, for

$$
\begin{gathered}
E_{2}=\left\{h_{1}, \ldots, h_{s}\right\} \subseteq\left\{h: h \subseteq A_{r}\right\} \\
\left|h_{k} \cap \bigcup_{1 \leqq i \leqq k-1} h_{i}\right| \leqq 1 \text { for } k=2, \ldots, s .
\end{gathered}
$$

Then

$$
c^{*}\left(H^{r}\right)=0 \text {. }
$$

Proof. We use the following theorem of Erdös [4]. There exists a function $f(t)$ so that if $n>N(t, c)$ and $e\left(G_{n}^{r}\right)>c n^{r}$ then

$$
K^{r}\left(\frac{t}{\mathrm{I}} \cdots, \frac{t}{r=1}, \frac{n}{f(t)}\right) \subset G_{n}^{r}
$$

Let $t=\max _{1 \leq i \leq r-1}\left|A_{i}\right|$. Suppose there exists an infinite sequence of graphs $G_{n}^{r}$ with $e\left(G_{n}^{r}\right)>c n^{r}$, for which

$$
H^{r} \mp G_{n}^{r}
$$

$$
\alpha\left(G_{n}^{r}\right)=o(n)
$$

Let $U$ be the set of vertices of a $K^{r}\left(t, \ldots, t, \frac{n}{f(t)}\right)$ contained by $G_{n}^{r}$, and $U_{r}$ $=\left\{x_{1}, \ldots, x_{l}, l=\frac{n}{f(t)}\right\} \subset U$ be the vertices in the $r$ th class. By (12), the subgraph of $G_{n}^{r}$ spanned by $U_{r}$ cannot contain a subgraph isomorphic to $L\left(A_{r} ; E_{2}\right)$.

Now we prove that by the condition (11) on $L\left(A_{r} ; E_{2}\right), G_{n}^{r}$, more exactly $U_{r}$. must contain a large independent set.

Let $H(j)(j=1, \ldots s)$ denote the subgraph of $H^{r}$ formed by the edges $\left(h_{1}, \ldots, h_{j}\right)$ and $G_{n}^{r}\left(U_{r}\right)$ denote the subgraph of $G_{n}^{r}$ spanned by the subset of vertices $U_{r}$. Suppose $H(k-1) \subset G_{n}^{r}\left(U_{r}\right)$ but $H(k) \sqsubset U_{n}^{r}\left(U_{r}\right)$ for some $1<k \leqq s$. Let $H_{1}$ be a copy of $H(k-1)$ contained in $G_{n}^{r}\left(U_{r}\right)$ and $V_{1}=V_{1}\left(H_{1}\right)$. By (11) there is a vertex $x \in V_{1}$ so that $x$ is independent of $U_{r}-V_{1}$. Note $\left|U_{r}-V_{1}\right|>n / f(t)-s r$. Now apply the same argument to $G_{n}^{r}\left(U_{r}-V_{1}\right)$. Thus, we obtain a vertex $x_{2} \in U_{r}-V_{1}$ which is independent of a set $U_{r}-V_{1}-V_{2}$ where $\left|U_{r}-V_{1}-V_{2}\right|>n / f(t)-2 r s$. We continue this process and obtain and independent set of size $\geqq n / f(t) r s$. Having (13) this contradiction proves the theorem.

Problem 1. Is condition $\left|h_{i} \cap h_{j}\right| \geqq 2$ in Theorem 1 necessary for the truth of (8)? 
Problem 2. Does there exist a graph $H_{n}^{r}$ for which

$$
0<c^{*}\left(H^{r}\right)<c\left(H^{r}\right) ?
$$

Problem 3. Let $V_{1}=\left\{x_{1}\right\}, V_{2}=\left\{x_{2}, x_{3}, x_{4}\right\}, V_{3}=\left\{x_{5}, x_{6}, x_{7}\right\}$, and $H^{3}$ be the hypergraph with $V\left(H^{3}\right)=\left\{x_{i}, 1 \leqq i \leqq 7\right\}$ and

$$
\left.E\left(H^{3}\right)=\left\{\left\{x_{2}, x_{3}, x_{4}\right\},\left\{x_{5}, x_{6}, x_{7}\right\} \text { and }\left\{x_{i}, x_{j}, x_{l}\right\}: x_{i} \in V_{1}, x_{j} \in V_{2}, x_{l} \in V_{3}\right\}\right\} .
$$

We know that $c\left(H^{3}\right)>0$. Is $c^{*}\left(H^{3}\right)=c\left(H^{r}\right)$ or $0<c^{*}\left(H^{3}\right)<c\left(H^{r}\right)$ or $c^{*}\left(H^{3}\right)=0$ ?

Problem 4. Let $V\left(H^{3}\right)=V_{1} \cup V_{2} \cup V_{3}$, where $V_{2}$ and $V_{3}$ are independent sets but the graph spanned by $V_{1}$ contains a circuit. What can one say on $c\left(H^{3}\right)$ ?

Problem 5. Is condition (11) in Theorem 2 necessary?

Problem 6. Find a function $h(n)$ so that

$$
\left(\begin{array}{l}
n \\
3
\end{array}\right)^{-1} f\left(n ; K_{n}^{3}, h(n)\right)=O\left(n^{3}\right)
$$

Our Theorem 1 gives that there is an $\alpha<1$ for which $f\left(n ; K_{4}^{3}, n^{\alpha}\right)>c_{2} n^{3}$ for a $c_{\alpha}>0$. Is inf $\left\{\alpha: \lim n^{-3} f\left(n ; K_{4}^{3}, n^{\alpha}\right)>0\right\}>0$ ?

\section{Graphs of uniform edge density}

Remark. We know that for every $\varepsilon>0$ there exists a graph $G_{n}$ so that $e\left(G_{n}\right)>$ $>(1 / 8-\varepsilon) n^{2}, K_{4} \oplus G_{n}$ and $\alpha\left(G_{n}\right)<\varepsilon n$. On the other hand, it is easy to see that if every subset of $V$ which is "small enough" has a not "too small" edge density then our $G_{n}$ must contain a $K_{k}$. Now we make this vague and heuristic statement more precise in two ways.

Proposition 1. Let $G_{n_{i}} ; n_{1}<n_{2}<\ldots$ be a sequence of graphs with the following properties:

$$
e\left(G_{n_{i}}\right)>c_{i} n_{i}^{2}
$$

(15) If $f$ is an arbitrary function with $\lim _{x \rightarrow 0} f(x)=0$ and if $i>i_{0}(\varepsilon)$ then in $G_{n_{i}}$ every set of $>\varepsilon n_{i}$ vertices' spans a subgraph of at least $f(\varepsilon) \varepsilon^{2} n^{2}$ edges. Then for $i$ large enough $K_{k} \subset G_{n_{i}}$.

Proposition 2. Let $k$ be given. For every $\varepsilon>0$ there is an $\eta=\eta(\varepsilon, k)>0$ so that if $G_{n}$ is a graph which satisfies that every subgraph of more than $\eta n$ vertices spans a subgraph of at least $\varepsilon \eta^{2} n^{2}$ edges, then $K_{k} \subset G_{n}$.

The proofs are easy and left to the reader.

Now we consider the analogous question for hypergraphs.

Concerning the case $r \geqq 3$ we have: 
Proposition 3. For every $\eta>0$ there exists an $\varepsilon>0$ and a graph $G_{n}^{3}$ having the following property

$$
\left.K_{4}^{3} \oplus G_{n}^{3} \quad \text { (and even more, } H^{3}(4 ; 3) \mp G_{n}^{3}\right)
$$

and each spanned subgraph of $G_{n}^{3}$ of more than nn vertices contains more than $\varepsilon\left(\begin{array}{c}\eta n \\ 3\end{array}\right)$ edges. of $i$, and

To see this, let $n=3^{k}, V\left(G_{n}^{3}\right)=\{1, \ldots, n\}, i=\sum \varepsilon_{v} 3^{v}$ the trinary expansion

$$
\begin{aligned}
& E\left(G_{n}^{3}\right)=\left\{\{i, j, l\}: i=\sum_{v=0}^{k} \varepsilon_{v}^{(1)} 3^{v}, j=\sum_{v=0}^{k} \varepsilon_{v}^{(2)} 3^{v}, l=\sum_{v=0}^{k} \varepsilon_{v}^{(3)} 3^{v},\right. \\
& \varepsilon_{v}^{(1)}=\varepsilon_{v}^{(2)}=\varepsilon_{v}^{(3)} \text { for } v<v_{0}, \quad\left\{\varepsilon_{v_{0}}^{(1)}, \varepsilon_{v_{0}}^{(2)}, \varepsilon_{v_{0}}^{(3)}\right\}=\{0,1,2\} .
\end{aligned}
$$

It is easy to see, that this graph has the above property. At the same time to every $\varepsilon>0$, there is an $\eta>0$ so that there is a spanned subgraph of $G_{n}^{3}$ of more than $\eta n$ vertices and contains less than $\varepsilon\left(\begin{array}{c}n n \\ 3\end{array}\right)$ edges. This means that the edge-density is not uniformly positive.

Problem 4. Assume now that we have an infinite sequence $\left(G_{n}^{3}\right)$ so that for every spanned subgraph of $m>\eta n$ vertices $G_{n}^{3}$ contains more than $c\left(\begin{array}{c}m \\ 3\end{array}\right)$ edges. Does it then follow that our graph contains $a K_{k}^{3}$ if $n>n_{0}(\varepsilon, c, r)$ ? We do not know the answer even for $k=4$, in fact, we do not even know whether our graph contains $a H^{3}(4 ; 3)$.

Perhaps it is clearer to state the problem in a sligthly weaker form.

Problem 5. Assume that $G_{n}^{3}$ has the property that every spanned subgraph of $m>\frac{n}{\log n}$ vertices contains at least $c\left(\begin{array}{c}m \\ 3\end{array}\right)$ edges. Does our graph then contain a $H^{3}(4 ; 3)$ or a $K_{4}^{3}$ or $K_{k}^{3}$ ?

The role of $\frac{n}{\log n}$ could be replaced by any $f(n)$ with $\frac{f(n)}{n} \rightarrow 0$.

Problem 6. Assume that there is a $c_{1}$ so that for every $x, y \in V\left(G_{n}^{3}\right)$

$$
\left|\left\{z:\{x, y, z\} \in E\left(G_{n}^{3}\right)\right\}\right|>c n .
$$

Is it then true that $H^{3}(4 ; 3) \subset G_{n}^{3}$ ?

Problem 7. We define a sequence of graphs $G_{n_{i}}^{3}(i=1,2, \ldots)$ to be uniformly distributed it for every $\eta>0$ there is a $c(\eta)$ so that for every $i>i_{0}(\varepsilon)$ every spanned subgraph of $m>\eta n$ vertices has $(c(n)+O(1))\left(\begin{array}{l}m \\ 3\end{array}\right)$ edges. Is there a graph $H^{3}$ so that there is an extremal graph belonging to $\mathrm{H}^{3}$ which is uniformly distributed?

We expect that such a graph does not exist. 


\section{References}

[1] B. Bol iobás and P. Ercós, On a Ramsey-Turán type problem, J. Comb. Th. Ser. B 21 (1976) $16 \epsilon-168$.

[2] P. Erdós, A. Hajnal, V. T. Sós and E. Szemeréd, More results on Ramsey-Turán type problems, Combinatorica, 3 (1) (1983).

[3] P. ERdös and A. HAJNAL, On chromatic number of graphs and set systems, Acta Math. Acad. Sci. Hungar. 17 (1966) 61-99.

[4] P. ERDös, On extremal problems of graphs and generalized graphs, Israel J. Math, 2 (1964) $183-190$.

[5] P. Erdős and V. T. Sós, Some remarks on Ramsey's and Turán's theorem. Comb. Theory and Appl. (P. Erdõs et al. eds.) Math. Coll. Soc. J. Bolyai 4 Balatonfüred (1969) 395-404.

[6] P. ERdös and M. H. Stone, On the structure of linear graphs, Bull. Amer. Math. Soc. 52 (1946) 1087-1091.

[7] V. T. Sós, On extremal problems in graph theory Proc. Calgary Internat. Conf. on Comb. Structures (1969) 407-410.

[8] E. SZEMERÉDI, On graphs containing no complete subgraph with 4 vertices (in Hungarian), Mat. Lapok 23 (1972) 111-116.

[9] P. TURÁN. Eine Extremalaufgabe aus der Graphentheorie (in Hungarian), Mat. Fiz. Lapok 48 (1941) 436-452. see also: On the theory of graphs, Colloquium Math. 3 (1954), 19-30.

\section{P. Erdős}

Mathematical Institute of the

Hungarian Academy of Sciences

Reáltanoda u. 13-15

Budapest 1053, Hungary
Vera T. Sós

Bell Laboratories

Murray Hill, NJ 07974, U.S.A. and Dept of Analysis $I$

Eörvös University

Múzeum krt. 6-8

Budapest 1088, Hungary 